\title{
Confining and compressing the atom ${ }^{\star}$
}

\author{
Jean-Patrick Connerade ${ }^{\mathrm{a}}$ \\ Quantum Optics and Laser Science Group, Physics Dept. Imperial College London and European Academy EASAL, Paris, \\ France
}

Received 27 July 2020 / Received in final form 1 September 2020 / Accepted 8 September 2020 Published online 13 October 2020

(C) The Author(s) 2020. This article is published with open access at Springerlink.com

\begin{abstract}
A review of effects due to the confinement and compression of atoms is presented. It is argued that they provide new insights into the properties of atoms and, in particular, into their interactions with the microscopic environment. Studies of confined atoms allow one to understand better the changes of behaviour which occur under pressure. Some applications to practical situations are also suggested.
\end{abstract}

\section{Introduction}

There exist fundamental connections between Thermodynamics and Quantum Mechanics which, in one way or another, are related to the problem of the Confined Atom. The Confined Atom is not usually approached from this point of view but, in fact, stands as one of the basic problems of physics which, somehow, has escaped attention for a long time. Often, it has been considered more as a curiosity than as a "real" problem, first, because it was not necessary to the development of Quantum Mechanics and second because, for a long time, even pioneers of the theory [1] found no convincing application of their results. More recently specific examples such as metalofullerenes have added substance to the problem, and the subject of confined atoms has been revived with much success. However, the connections to fundamental physics remain relatively undocumented. The present paper attempts to fill this gap by pointing out how close confined atoms are to some standard problems in the development of modern physics.

\section{Thermodynamics and quantum mechanics}

Fundamental connections between Thermodynamics and Quantum Mechanics emerge at several levels as both theories are developed. To set the scene, it is useful to begin by recalling briefly some salient examples.

First, we have the "UV catastrophe". Without Quantum Mechanics, one cannot make sense of the properties of Black-Body radiation, which provide a basic

* Contribution to the Topical Issue "Atoms and Molecules in a Confined Environment" edited by C.N. Ramachandran, Vincenzo Aquilanti, Henry Ed Montgomery, Narayanasami Sathyamurthy.

a e-mail: jean-patrick@connerade.com measure of temperature in Thermodynamics. The discrepancy between Classical Physics and Quantum Physics is greatest at the high energy end of the Black-Body spectrum, and the necessary correction to the Rayleigh-Jeans theory provides the very basis for the introduction of Planck's constant [2].

Second, we have the anomaly of specific heats, raised by Dirac [3]. If Classical Physics were strictly applied, the dynamics of the universe should be infinitely divisible into more and more component parts within a smooth and continuous space, as first imagined by Pascal [4]. In this model, all specific heats should be infinite and the equipartition theorem would be impossible to apply. The reason this does not occur is again due to quantum mechanics. There is no infinite divisibility of space or of the component parts of any mechanical system by virtue of the Uncertainty Principle.

Third, as Statistical Mechanics was developed, a problem emerged for which an empirical "fix" was adopted in the definition of the entropy, to resolve the so called "Gibbs paradox" [5]. A factor $1 / \mathrm{N}$ ! introduced to remove this unwelcome effect was at first justified simply because it gave the right result. It was only found later that this factor follows from another remarkable property of Quantum Mechanics: the indistinguishability of identical particles, introduced by Pauli [6].

Finally, there is the connection between Quantum Mechanics and the emission of radiation of temperature $T$ from a system with quantised energy levels and a population of states described by Boltzmann's law which enabled Einstein $[7,8]$, by using his approximation of "twolevel atoms" and the Principle of Detailed Balance, to recover Planck's black-body radiation formula through the introduction of the spontaneous emission coefficient $A_{21}$. This is a remarkable connection, because the existence of the spontaneous emission coefficient results from second quantisation, but was actually discovered by involving Thermodynamics. It is noteworthy, when the resulting 
Einstein-Milne relations [8] are applied to the optical pumping of a laser, that at least three levels are required, since order can only be created at the expense of energy, in accordance with the Second Law of Thermodynamics.

From all these examples, it is apparent that the simultaneous application of principles drawn from the Quantum Theory on one hand and from Thermodynamics or Statistical Mechanics on the other has often proved useful to the development of theoretical physics.

\subsection{Thomas-Fermi theory and atomic confinement}

By calling on Statistical Mechanics and on some aspects of Quantum Mechanics, Thomas [9] and Fermi [10] independently developed the Thomas-Fermi model of the atom. Key assumptions are (i) that the electrons of a manyelectron system can be treated as a thermodynamic "gas" (ii) that two electrons can be lodged in every volume $h^{3}$ of phase space and (iii) that, with this prescription, the electrons completely fill the whole of the Fermi sphere up to energy $E_{\mathrm{F}}$ and momentum $p_{\mathrm{F}}$. Within this scheme, the charge density can be expressed via statistical theory and, independently, via Gauss's theorem. The two equations, solved simultaneously, yield the second order non-linear Thomas-Fermi differential equation:

$$
\mathrm{d}^{2} \varphi / \mathrm{d} z^{2}=\varphi^{3 / 2} / \operatorname{sqrt}(z)
$$

where $\varphi(z)$ is a dimensionless potential, defined through $\mu-V(r)=\left(Z e^{2} / r\right) \varphi(r / b), \mu$ is the chemical potential (introduced via a Lagrange multiplier) $V(r)$ is the electrostatic potential and the constant $b=(1 / 4)\left(9 \pi^{2} / 2 Z\right)^{1 / 3} a_{0}$, while $a_{0}$ is the Bohr radius and $Z$ the atomic number. It is convenient also to define a dimensionless length $z=r / b$

The solutions $\varphi(z)$ are of three kinds, illustrated in Figure 1. For $\mu=0$, the charge density is everywhere non-zero and $\varphi(r)$ tends to zero at infinity (solution $b$ ), which describes a neutral atom. For $\mu<0$, we obtain an ion, with positive overall charge at the centre (solution $a$ ), a negative charge cloud within a finite radius and a system "edge" at $\varphi\left(r_{a} / b\right)=0$. For $\mu>0$ (the case of interest to us here, viz. solution $c$ ) the system is "compressed" by a rising repulsive potential at finite radius, i.e. the negative charge is squeezed into a small space, and an "edge" of the system appears at a radius $r_{c} / b$, where $d \varphi / d z=\varphi / z$.

Because of a neutral charge distribution and a steadily rising potential as $r_{c}$ is approached, the third type of solution is interpreted as describing a "compressed atom". Thus, the Thomas-Fermi $(T-F)$ model naturally provides a simple example of a spherically confined atomic system.

The $T-F$ approximation is inadequate in many respects. It does not account for exchange and does not yield the shell structure of the atom correctly.

Mathematically, it can be put on a quite rigorous footing. Dirac [11] introduced the exchange term and the theory has been much refined since [12], leading also to the development of Density-Functional Theory [13]. This being said, the $T-F$ scheme provides an instance of confinement for electrons similar to the ideal gas of Thermodynamics. We thus form a picture of the confined atom as

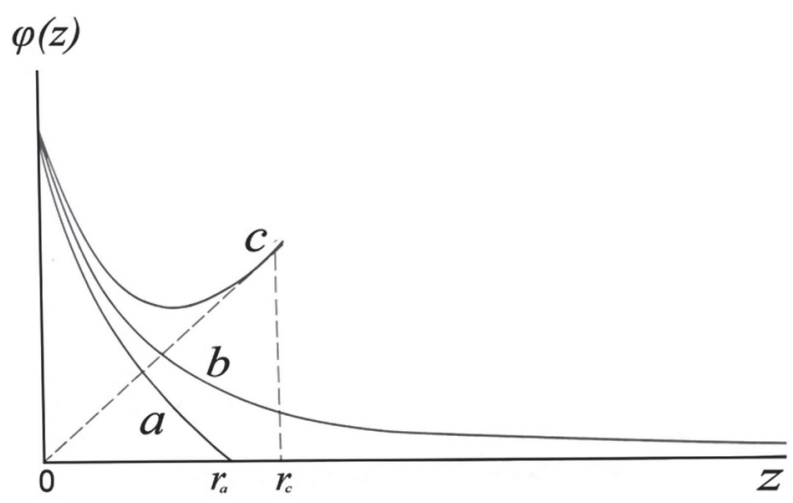

Fig. 1. The three types of solution of the Thomas-Fermi model of the atom $(a, b$ and $c)$. The solution labelled $c$ is the one of interest here.

an electronic system which can itself be compressed, either by increasing the charge at the centre, or by introducing a potential "step" at the edges. It can be instructive to compare theoretical results from both methods of compression (internal and external) [14]. At the surface of the Fermi sphere, the highest occupied states possess a Fermi momentum $p_{\mathrm{F}}$. Some authors introduce a Fermi temperature $T_{\mathrm{F}}$ by:

$$
E_{\mathrm{F}}=p_{\mathrm{F}}^{2} / 2 \mathrm{~m}=\mathrm{k} T_{\mathrm{F}}
$$

where $k$ is Boltzmann's constant (see e.g. [15]). It is somewhat artificial to introduce a temperature in this way. A consistent definition of temperature in Quantum Mechanics requires either a Boltzmann or a Fermi-Dirac distribution of populations in the microstates. This is definitely not the case in the $T-F$ scheme, which really describes a zero temperature system, with all accessible states filled up to the level $E_{\mathrm{F}}$, and all states above $E_{\mathrm{F}}$ empty. Thus, there is no thermal agitation to justify introducing a physical temperature. Referring to the expression for an increase in internal energy in Thermodynamics:

$$
\mathrm{d} U=T \mathrm{~d} S+P \mathrm{~d} V
$$

we should set $T=0$ for the $T-F$ atom, so there can be no contribution from the first term. However, the volume of the atom under pressure is well-defined and depends on applied pressure, so the second term in (3) does indeed contribute. This being said, the $T-F$ model does not account for periodic properties of atoms (and size) correctly as it predicts a radius only weakly dependent on atomic number $Z$.

\subsection{Confined hydrogen from the Schrödinger equation}

As the above example shows, confinement is naturally introduced "from the outside" via external boundary conditions, i.e. the outer reaches of the potential act like a "classical piston" compressing the system.

In principle, this mode of compression can be applied to any atomic model, but how to achieve it in a very simple way from the Schrödinger equation was first discovered almost accidentally in a rather speculative theoretical paper. Sommerfeld and Welker [1], a number of years 
after the Thomas-Fermi model and its solutions had been published, made this essential contribution. They were studying regular $\ell=0$ solutions of the Schrödinger equation for the free atom hydrogen. They noticed that, since the solutions have nodes which recapitulate, placing an infinitely high repulsive wall at the radius of any node allows one to satisfy usual boundary conditions exactly. Thus, applying the theorems of Quantum Mechanics, one recovers the exact, $\ell=0$, solutions to another problem: the hydrogen atom confined within a spherical square well of appropriate radius, with infinitely high repulsive walls. The solutions are simply the inner loops of the hydrogenic wavefunctions for the specific confining spheres. Furthermore, since an infinite number of nodes (and appropriate confining spheres) exist for the ideal free atom, the general solution for any radius of confinement can be expanded as an infinite series formed from the complete set of solutions. At first sight, Sommerfeld and Welker had given the full solution to the quantum mechanical problem of hydrogen confined inside a hard spherical cavity (a point to which, however, we will need to return to below).

A further study of the properties of confluent hypergeometric functions applied to compressed hydrogen was reported by De Groot et al. [16]. A more recent review, for both confined hydrogen and confined helium has been presented by Aquino [17].

\subsection{The meaning of the Sommerfeld and Welker solutions}

The problem solved by Sommerfeld and Welker [1] seemed to have no application to any real physical system at the time. The concept of "hydrogen within a sphere" was of no particular experimental significance. In their original paper, they attempted to account for the delocalisation of electronic wavefunctions in solids as a consequence of confining an atom in a box (the unit cell of a crystalline solid) but this explanation lacked conviction: spherical confinement is of course rather different from confinement in a unit cell, which is a box with at least cubic (and usually, more complex) shape and communicates with a lattice. The issue was taken up again by Michels et al. [18] who suggested that the Sommerfeld-Welker theory is more appropriate to discuss hydrogen atoms under pressure. An interesting remark had already been made by Sommerfeld and Welker themselves. They had found that the binding energy of the electron decreases with increasing confinement until it is effectively "ionised", i.e. is no longer bound by the electrostatic field of the nucleus, but directly confined by the surrounding spherical potential. This was the basis for their application of the theory to the localised/itinerant transition in a conducting solid, although, of course, they could provide no description of the lattice using such a simple idea.

\subsection{Why study pressure effects in heavier atoms?}

Although studies of light atoms provide simple examples of the processes involved in atomic confinement, they have

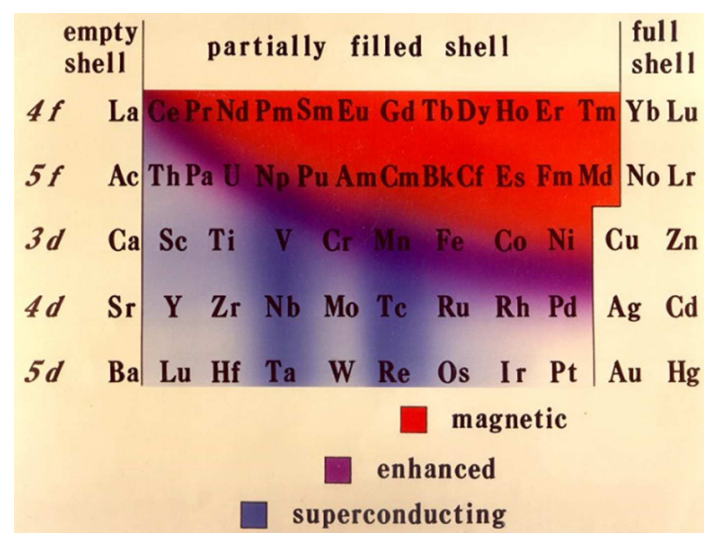

Fig. 2. The "Quasi-Periodic" Table of Smith and Kmetko (see text). Note that the $d$ sequences are spread out wider than the $f$ sequences.

limited application. Light atoms, in fact, change little under compression. To take the case of helium, it has the highest ionisation potential in the Periodic Table and is therefore the most rigid of all the elements. At the opposite extreme, compressing lithium acts mainly on the outermost electron and reproduces the features of confined hydrogen without involving the inner closed shell, because the energy required to "break it open" is so much higher than the first ionisation potential.

The confinement of atoms in the first three rows of the Periodic Table does not induce significant changes in chemical properties, such as valence, but mainly induces differences of electronegativity. A general description of the behaviour of confined atoms is given in the review by Jaskólski [19]. The effects of pressure become much more significant as one moves down in the Periodic Table towards transition elements and the rare-earths.

In Figure 2, we show an outline of results obtained experimentally in the context of studies of magnetism and conductivity by Smith and Kmetko at the Los Alamos laboratory [20]. These researchers studied the boundary between localised and itinerant behaviour in solids and compounds containing many different atoms in the sequences corresponding to the $d$ and $f$ transition elements. Their conclusions inspired them to draw up a 'quasi-Periodic Table' summarising properties, which they correctly inferred to be of atomic origin.

The purpose of their table is to display a "ridge" which sweeps diagonally from La to Pd and separates conducting elements (with delocalised electrons) from magnetic elements (with localised electrons). The localisation to delocalisation transition is referred to in condensed matter physics as a first order Mott transition. In Atomic physics, the interpretation of the table is different. We recognise the elements along this ridge as those which exhibit prominent "giant resonances". Solids and compounds formed from these elements exhibit pressure sensitivity. Ce, for example, makes a transition from the $\alpha$ to the $\gamma$ phase under pressure, with a sudden change in volume and in the speed of sound, U becomes superconducting under pressure, etc. [20]. Chemists will also recognise this table as 


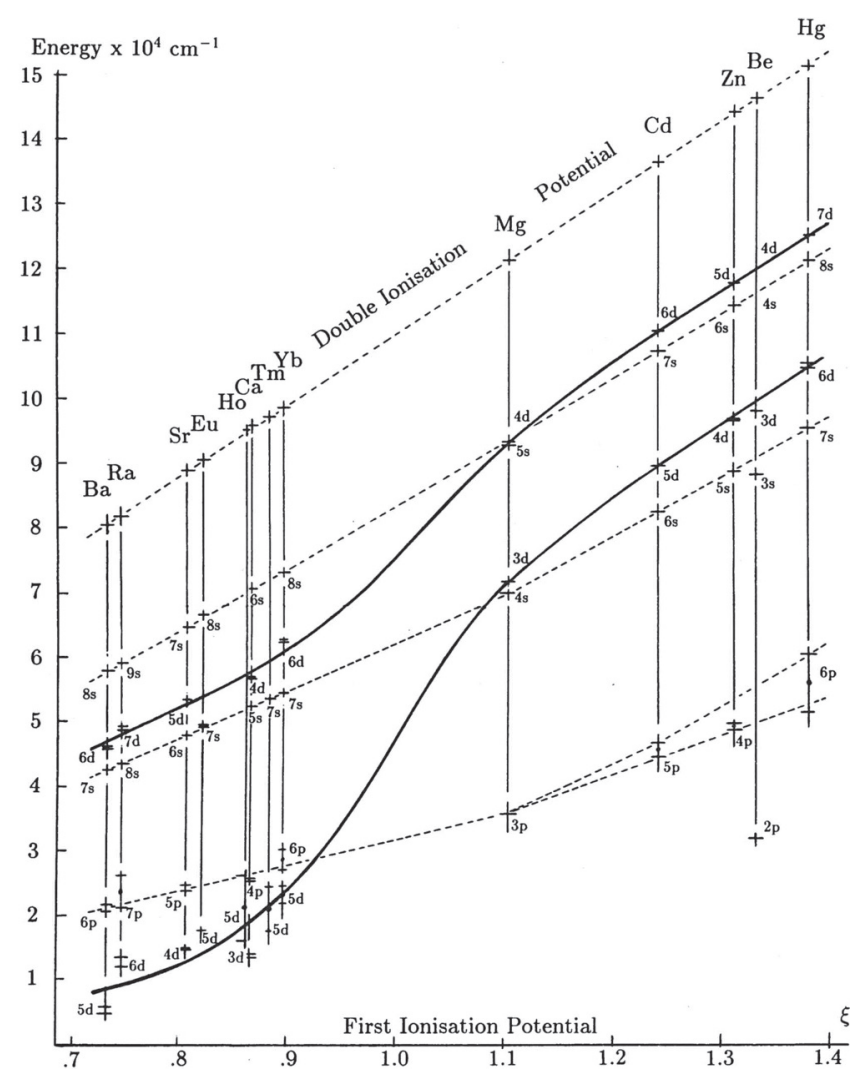

Fig. 3. The effects of $d$-orbital collapse and consequent $s-d$ competition can be displayed from experimental data by plotting level diagrams along a homologous sequence of atoms and ions in terms of a dimensionless parameter (see [28] for details). Such plots reveal the progressive level crossings of the full $s$ and $d$ manifolds as a function of the effective central charge in the system.

regrouping many useful elements for hydrogen or $\mathrm{Li}+$ ion storage, and also elements susceptible to instabilities of valence or playing a significant role as catalysts $(\mathrm{Pd}$, in particular).

All of these characteristics, as will emerge below, are related to the high atomic compressibility of $d$ and $f$ orbitals which makes elements close to the ridge in Figure 2 particularly "soft".

\subsection{High atomic compressibility}

The underlying cause of the long periods in Mendeleev's Table was first suspected by Fermi [22] who realised that the accepted explanation [24] as a simple consequence of electrostatic "screening" is inadequate. This explanation would require a new kind of force in physics, acting on some electrons but not on others. By considering the radial Schrödinger equation, rather than merely the angular properties invoked by Bohr and Stoner [23], Fermi realised that the centrifugal term in the effective radial potential - via the $\ell$-dependent repulsive term $\ell(\ell+1) \hbar^{2} / 2 m r^{2}$ - would act differently on electrons of different angular momentum. To confirm his hypothesis, Fermi entrusted

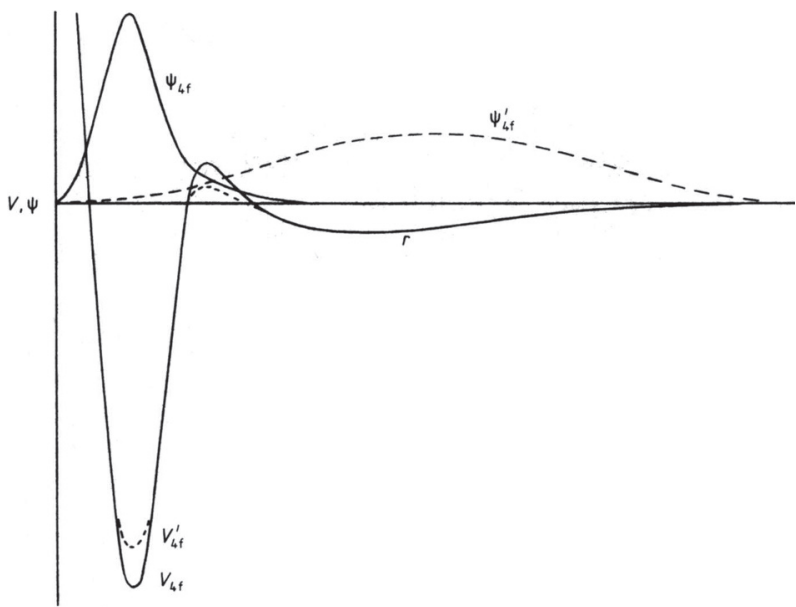

Fig. 4. (Schematic) A deep inner well develops in the effective $f$-radial potential of atoms around $Z=57$. For a very small variation in its parameters, it can suddenly satisfy the quantum condition to support a bound state and the solution flips from the outer coulombic well to allow $4 f$ deep shell filling.

his co-worker, Maria Goeppert-Mayer, with calculating from first principles whether the effect of the centrifugal term might suffice to explain the breakdown of the aufbau or "building-up" principle around Ca.

Unfortunately Goeppert-Mayer [25] (for an obvious reason) went about her task using the Thomas-Fermi model which, as noted above, does not account correctly for the shell structure of the atom. She was able to verify Fermi's hypothesis for the very strong effect which indeed occurs at $\ell=3$ (the $f$-subshells - lanthanides and actinides) but the explanation failed for $\ell=2(s-d$ competition in the transition elements). Thus, her results were, at least in part, inconclusive and the "official" explanation as a pure screening effect stood unchanged until a more realistic atomic model based on the Hartree-Fock method was applied to the problem [26]. It then became apparent that Fermi's initial hypothesis had been completely correct and that the effect is quantum mechanical in origin [27].

As guessed by Fermi and clearly revealed by experimental data, the centrifugal barrier term acts selectively on $d$ orbitals but not, of course, on $s$ orbitals, (see Fig. 3).

The revised calculations [26] confirmed the influence of a repulsive potential barrier which initially "pushes out" electrons of $\ell=2$ and 3, inhibiting the filling of the $d$ and $f$ subshells. However, as the atomic number $Z$ increases, a potential well inside the atom appears, deepens more and more, and eventually "pulls in" the electron. One thus has either competition with $s$-subshell filling (in the case of $d$ electrons) or the sudden filling of a new subshell deep inside the atom (for $f$-electrons) referred to as orbital collapse.

The double-well effective potential which causes the lanthanide sequence was found by Goeppert-Mayer [25]. A "knife-edge" situation develops towards $\mathrm{Ba}$ and La. It requires only a slight alteration of the balance between an inner and an outer well in the atom (see Fig. 4) for the electron involved to migrate suddenly from one well 
to the other. An atom of this type placed inside a sphere is anomalously "soft" and will respond very markedly to changes in external pressure. There is even the possibility of fundamentally changing its electronic configuration, its valence and all of its chemical properties. Atoms with such extreme sensitivity are obviously of interest from the standpoint of the energy variational principle.

\subsection{Spherical confinement and the Self-Consistent Field}

To illustrate the effect just described close to the onset of filling of the $3 d$ subshell (at $\mathrm{Ca}$ ) we show the result of compression on the $3 d$ orbitals around $Z=20$ in Figure 5 [14].

The figure has two parts, each one corresponding to a method of compression in Section 2.1, viz. (a) compressing the atom by a "spherical piston" (different radii for this sphere are indicated alongside the different solutions obtained) (b) artificially increasing the charge on the nucleus, which has a similar effect. We describe these different methods of compression as $(a)$ external and $(b)$ internal.

While it is feasible to encapsulate an atom within spheres of different sizes (for example: within various hollow molecules), increasing the charge at the centre by non-integral amounts is not achievable experimentally. However, this is sometimes a useful theoretical device to probe the effects of compression or of insertion into a solid state environment.

In order to probe atomic compressibility in the range of greatest interest, it is important to extend the treatment of the atom confined in a sphere to elements from the long periods of the Periodic Table and, specifically to those in the table of Smith and Kmetko [20]. This opens the way to probing the mechanisms involved in $s-d$ competition directly and to understanding differences of chemical behaviour for atoms under high pressure as compared to their more familiar properties at normal pressures.

\subsection{The periodic table under pressure}

The behaviour of the Thomas-Fermi atom under pressure (solution $c$ of Fig. 1 above) provides a method to set up the same problem with a more realistic atomic model (for example, Hartree-Fock). We can calculate how the selfconsistent field is altered for a many-electron atom contained inside a sphere. It is sufficient to introduce a step in the outer reaches of the potential and then solve the selfconsistent field equations within the sphere. An extreme approach (hard spherical containment) is to follow Sommerfeld and Welker and apply a very high repulsive step as the external boundary condition. Confinement introduced in this way acts like the "classical" piston used in thermodynamics to compress an ideal gas. It is also similar to confining the electron gas (as in the $T-F$ model) except that, in this case, the compressed medium is composed of atomic shells with magic numbers which alter their compressibility as compared to a truly fluid medium.

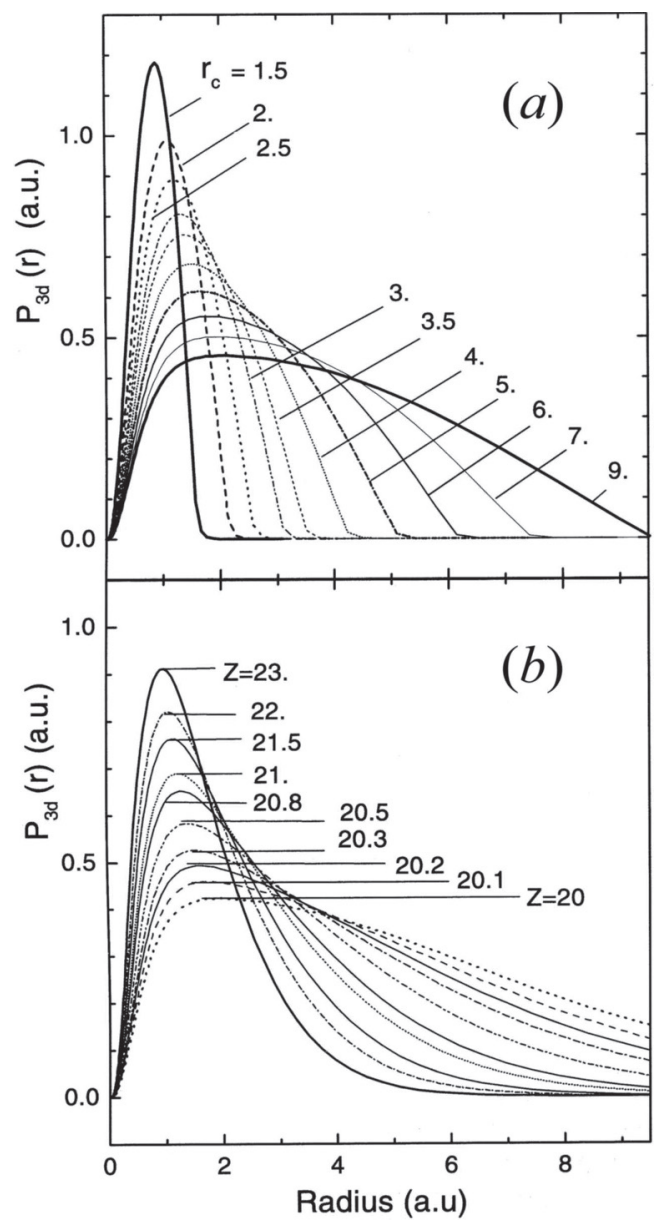

Fig. 5. The influence of compression on the $3 d$ wavefunction of Ca as obtained $(a)$ by external confinement within a sphere and $(b)$ by increasing the charge on the nucleus (see text for details). [14] Note the similarity between the wavefunctions in the inner reaches of the atom.

Subshells which are especially "soft", of course, react differently to external pressure from those which are more rigid. When compressed, they flip inside the other shells and their binding energies can also cross over and fall below the binding energies of neighbouring shells.

Compression can therefore reverse the behaviour described as " $s-d$ competition", which occurs in the filling of the long periods beyond $\mathrm{Ca}$ in the Periodic Table. The filling of shells is then altered under pressure for elements of the $3 d, 4 d$ and $5 d$ periods. Specifically, the order of filling is changed at high pressures, with $3 d$ filling occurring before $4 s, 4 d$ before $5 s$ and $5 d$ before $6 s$, as would have been predicted by continuing to apply the aufbau principle blindly to the elements beyond $\mathrm{Ca}$.

Thus, the Periodic Table for elements under high pressure is not at all the same as for free atoms [29]: the outermost electronic configuration, the valence and the binding energies are all different. This explains why the chemistry of elements in this part of the Periodic Table is transformed at very high pressures.

In reference [29] the influence of high pressure on the filling of shells in the Periodic Table is explored within 
a simple self-consistent field atomic model and a general conclusion is drawn: with increasing pressure, the $d$ and $f$ shells fill earlier and, ultimately (i.e. at very high pressures), the order of filling predicted by the Aufbau Prinzip is recovered without the anomalies of Mendeleev's Table at ordinary pressures. In fact, as the applied pressure is increased, atoms move away from anomalies of filling and tend towards the simple ordering of the elementary rules.

In addition to the bound states of the inner short range wells of the effective $d$ and $f$ potentials, a number of resonances exist (the "giant resonances" [30]) which are beyond the scope of the present review. They are found to obey the rules of Levinson's theorem in scattering theory [31] in that the number of resonances up in the continuum relates to the number of bound states of the well, although it would be very difficult to prove such a theorem for the case of an internal short-range potential coupled to an external asymptotic Coulomb well [32].

Regarding the filling of shells and orbital collapse (both $s-d$ and $d-f$ competition), independent supporting evidence comes from a recent paper [69].

\subsection{Relativistic effects}

There is a difficulty to resolve as one moves towards heavier elements. It concerns the influence of relativity. In general, Dirac-Fock calculations show [33] that the main effect of relativity is to modify the compactness and diffuseness of wave-functions as compared to the non-relativistic solutions. For this reason, simulating relativistic effects indirectly, merely by introducing a pseudopotential (as is frequently done for energy level determinations to avoid the complex machinery of the Dirac equation) is not the best option. This is especially true for confined atoms in the long sequences, the lanthanides being particularly critical. Since these atoms are very "soft", small changes in energy lead to large changes in the radial wavefunctions and relativistic effects must be incorporated accurately. For this reason, some effort has been devoted [33] to the challenging problem of atomic confinement within the Dirac-Fock scheme.

It turns out one has to be rather careful not to apply Sommerfeld and Welker boundary conditions to the fully relativistic atom. The large and small components of the relativistic spinor can only be zero simultaneously at the origin $(r=0)$. If one attempts to force them both to zero at another radius (the position of the confining sphere, for example) then the "step" in energy required is much larger than twice the rest mass of the electron. The apparent consequence (sometimes called Klein's paradox) would be the spontaneous creation of electron-positron pairs at the confining "edge", which of course does not occur. Formally, there are different kinds of boundary conditions one can apply to "contain" Dirac electrons [34], but the simplest practical solution to the problem is to use a finite step in potential just high enough to "hold", say, 99\% of the large component of the relativistic spinor inside the sphere, thus allowing a very small quantum-mechanical penetration of the confining "piston". This is not only the easiest method to apply numerically. It is also closer to

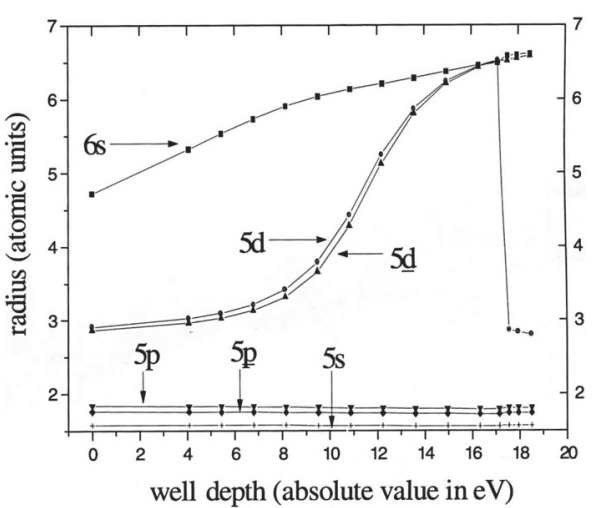

Fig. 6. The mean radius of $5 s$ to $6 s$ orbitals in @La plotted against the well depth of an attractive confining shell [33].

the physical situation for "real" containment of an atom than the unrealistic assumption of an infinitely high step in the confining potential.

It is also possible to confine atoms inside a spherical attractive shell rather than a repulsive piston, in which case orbitals will be dilated rather than compressed. This occurs, for example, when an atom is confined endohedrally (i.e. inside) a fullerene molecule. For a metal atom $\mathrm{M}$, we refer to the combined system as a metallofullerene: $\mathrm{M} @ \mathrm{C}_{60}$.

In Figure 6, we show an example of Dirac-Fock calculations for La@ $\mathrm{C}_{60}$. The calculations [33] are performed for various well depths, and so a well depth of $0 \mathrm{eV}$ (at the left of the figure) corresponds to the free atom, which has one $5 d$ electron more tightly bound than the two $6 s$ electrons. Hovever, this $5 d$ electron lives in a very soft potential, just like the $3 d$ electron of $\mathrm{Ca}$ in Figure 5 above, so when placed in an attractive external shell, it can be progressively "sucked out" from the atom and eventually, the $6 \mathrm{~s}$ electrons "fall in" to occupy its original radius. This is an example of how " $s-d$ " competition in a neutral atom can be "reversed" by an external spherical perturbation.

\subsection{The quantum-mechanical "Piston"}

If we consider a more realistic situation than the "hard" piston (an atom trapped inside a hollow molecule, for example) then it becomes apparent, not only that there must be "leakage" of the atomic wavefunction into the world outside but also that the solution devised by Sommerfeld and Welker [1] actually misses a very important aspect of the whole problem. The external boundary conditions of the Schrödinger equation correspond to the "classical" (large $r$ ) reaches of the atom. If one begins to introduce confining "steps" or to impose boundary conditions which force the wavefunction to zero at a finite radius, then the question of the "quantum size" of the confining cavity arises. For cavities of very large size compared to the atom, the treatment adopted by Sommerfeld and Welker [1] is allowable in the limit. However, if the size of the cavity becomes small (i.e. commensurate with the size of the confined atom), the treatment is incomplete. 
It then becomes necessary to consider the confining cavity itself as an object with quantum properties (for example, a spherical square well in elementary Quantum Mechanics). Under these conditions, the confining cavity itself possesses resonances, independent and additional to those of the confined atom. In other words, the cavity itself has a spectrum, which mixes with the spectrum of the confined atom. The quantum states of the cavity are missing from the treatment of Sommerfeld and Welker [1]. The problem they have solved is the confinement of hydrogen within a classical cavity, but this ceases to be the full story for a cavity of quantum size.

It is an interesting property of the Schrödinger equation that it describes the quantum properties of a system, but normally makes use of external boundary conditions extending smoothly at large radius into the classical domain. If, as suggested by Sommerfeld and Welker, we place a high "wall" at the node of a wavefunction, we may satisfy the boundary condition at that point, but the consequence is the introduction of quantum states which cannot be neglected. The wall must be infinitely high and repulsive for the boundary condition to be strictly satisfied by forcing the atomic wavefunction to zero. Such a system (an atom confined within impenetrable walls) cannot truly exist because it is unobservable, which violates basic principles of Quantum Mechanics. To make the cavity "real" the confining walls must be of finite height, so that the atom can communicate with the outside world. It will then interact with the resonances of the cavity and the wavefunction is not simply the inner loop of a hydrogenic solution.

The Sommerfeld and Welker problem is of course completely idealised (even more so than atomic hydrogen itself in absence of any radiation field). Not only it cannot radiate but it cannot ionise. In fact, nothing can escape from the confined system which, in effect, cuts off its content from the outside world. The problem is useful to consider because it can be solved exactly, but one has to be careful how its features are compared with experiment.

\subsection{The concept of "Atomic Pressure"}

By convention, in classical thermodynamics one refers to the kinetic theory of gases to "explain" the concept of pressure: it is considered as an average force exerted on the walls of a vessel containing the gas by the impacts of atoms or molecules, treated as point particles. In this description, the atom is naturally incompressible.

The situation is altered by the introduction of the van der Waals corrections to the ideal gas law, one of which involves subtracting from the molar volume of the gas the volume occupied by one mole of the constituent molecules themselves, no longer considered as point particles. In van der Waals' theory, this volume is still regarded as a constant but, from the moment it is introduced, the possibility exists that the volume occupied by the atoms or molecules should itself depend on the pressure. Thus, the definition of pressure must itself be refined, and the medium to which it applies is no longer simply an assembly of point particles.

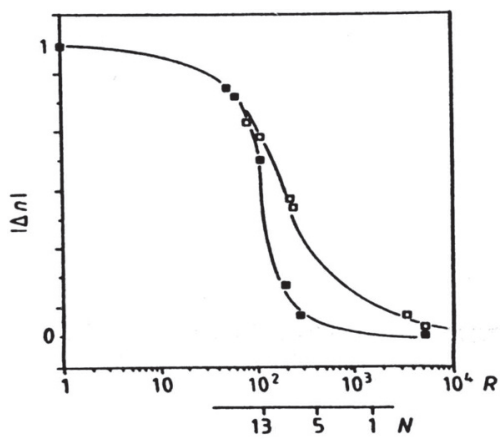

Fig. 7. The valence change $\Delta N$ of $\operatorname{Sm}(\boldsymbol{\square})$ and $\operatorname{Tm}(\square)$ clusters as a function of the number of atoms $N$ estimated from EXAFS data [38] showing how the size-dependence of cluster pressure precipitates a sudden change for clusters of both metals.

In fact, "pressure" acquires several new meanings when applied to molecular systems. We have the "chemical pressure" [35] which relates, for example, to the substitution of an intruding constituent in a crystalline lattice structure. As a consequence of Quantum Mechanics, one can define the Hellman-Feynman pressure $[36,37]$ involving the force exerted on an atomic nucleus by the electrons under an infinitesimal displacement.

Many kinds of pressure are also exerted on an atom by its environment. A beautiful example occurs in cluster physics as a function of the "size" of a cluster, (i.e. the number of atoms in the cluster), because of the difference between surface and bulk properties.

As discussed above, atoms from the lanthanide sequence in the Smith and Kmetko table are particularly sensitive to small changes in local pressure which disturb the balance between inner and outer reaches of the $f$-potential. A fine example is the valence change in Sm and Tm metal clusters, illustrated in Figure 7 [38].

The concept of an "atomic pressure" computed directly from the Hartree-Fock equations was introduced in [39] to explore the connection between intermediate valence and valence instabilities on the one hand (such as those of Fig. 7) and atomic orbital collapse on the other. Although such instabilities in rare-earth compounds are usually regarded as a theme of research of solid state physics [40] the various models used ([41-44], etc.) involve screening parameters which add to the complexity of the problem and to uncertainties about the driving mechanism, in particular to the relative magnitudes of lattice and electronic mechanisms [40]. Typical examples include the isomorphic phase transitions observed in solid Ce, in $\mathrm{SmS}$ and their alloys. By calculating the pressure required to flip a single element around $Z=56$ from having the $4 f$ electron in the outer reaches to having it trapped in the inner well of the $f$-potential, a figure of 790 bar is obtained without any adjustable parameters, directly from the Hartree-Fock equations [39]. By comparison, the $\alpha$ to $\gamma$ phase transition in $\mathrm{Ce}$ and its compounds is driven on a scale of kilobars and the SmS lattice collapse (see Fig. 1 in [39]) occurs at $6 \mathrm{kbar}$, within an order of magnitude of the Hartree-Fock estimate for the free atom. 


\subsection{Systematic trends in atomic compressibility}

In addition to the centrifugal barrier of $d$ and $f$ electrons, the shell structure of the atom plays a key role in determining pressure effects: the pressure required to force-ionise a large alkali atom with a low ionisation potential such as Cs is obviously very much lower than to break open the closed shell structure of He, with the highest ionisation potential of the Periodic Table.

A systematic study of the scaling laws for atomic compressibility to compare these extremes of behaviour is reported in [45]. All the calculations were performed under spherical compression, both internal and external, within a single-configuration Dirac-Fock scheme [46] modified to treat spherical confinement [47].

The idea is to place all atoms on a similar scale by defining, first, a dimensionless "shrinkage parameter"

$$
\xi \equiv \mathrm{V}^{p} / V^{f}
$$

where $V^{p}$ is the volume of the atom (obtained from the computed average radius) at a pressure $P$ and $V^{f}$ is the atomic volume of the free atom. This procedure scales out the intrinsic difference in size between, say, helium and caesium.

Next, we define a reduced energy:

$$
\varepsilon \equiv \mathrm{E}_{B}^{p} / E_{B}^{f}
$$

where $E_{B}^{f}=E_{a}^{f}-E_{i}^{f,} E_{a}^{f}$ is the total energy of the ground state of the free atom and $E_{i}^{f}$, of the free ion. In the same way, $E_{B}^{p}=E_{a}^{p}-E_{i}^{p}$, where the superscript $p$ refers to the atom and ion under a pressure $P$. Finally, we appeal to the familiar thermodynamic relations to define a "quantum pressure":

$$
\begin{aligned}
P & \equiv-\Delta E / \Delta V=-\left(E_{B}^{f} / V^{f}\right)(\varepsilon-1) /(\xi-1) \\
& =-\left(E_{B}^{f} / V^{f}\right) \oint=-\eta \oint
\end{aligned}
$$

where $\delta$ is a reduced pressure and $\eta$ is the atomic scaling factor.

In practice, when using ab initio theory to determine $\varepsilon$, it proves simpler and more consistent to use the Koopmans removal energy for each of the Dirac-Fock orbitals to replace the energies $E_{B}^{f}$ and $E_{B}^{p}$ [35]. This approximation is valid only if the atomic core is hardly perturbed under internal and external compression, and this turns out to be true: the inner shells are quite rigid compared to the valence shells.

Following through these calculations, one finds that Cs is roughly 2000 times more compressible than He but, despite this enormous difference, it turns out that the reduced compressibility, or $(\xi, \varepsilon)$ curves, are remarkably similar for both atoms (see Fig. 8).

A simple interpretation of this similarity is that the electronic cloud in atoms is held together by a balance of coulombic forces. Once the intrinsic size of the system is properly scaled out, what remains differs only from atom to atom by screening effects in the atomic potential.

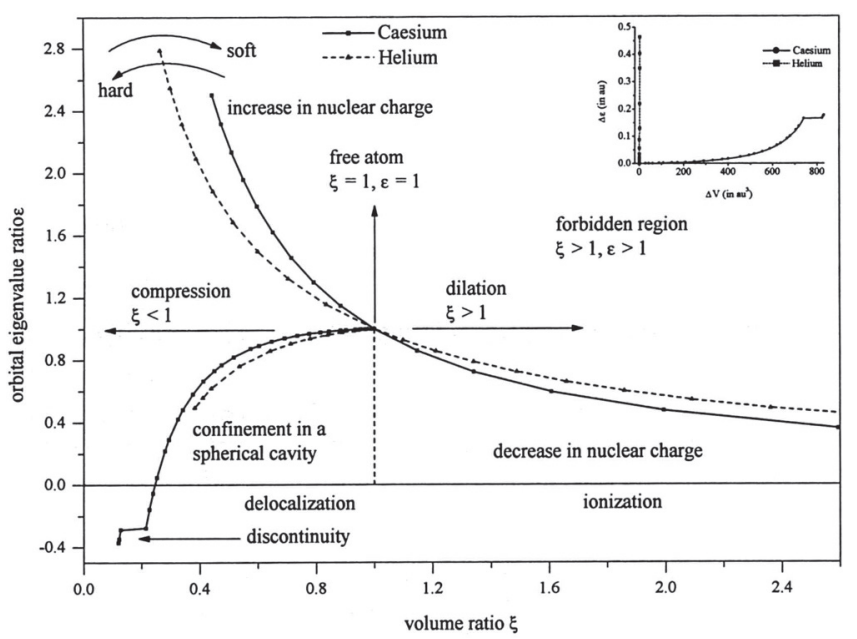

Fig. 8. Reduced compressibility plots for the atoms Cs (solid curve) and He (dashed curve). Two branches occur, one for internal compression by progressive ionisation and one for compression by an external impenetrable spherical "piston". They meet at the $(1,1)$ free atom point. Dilation can also be tracked as nuclear charge is decreased. In the inset, we show real compressibility (unscaled) for Cs. The discontinuity in the curve is not an artefact. It is due to orbital collapse in Cs [48].

The following set of general rules apply to the $(\xi, \varepsilon)$ curves of Figure 8

(a) as the effect of the spherical piston tends to zero, all such curves go through a common point $(1,1)$ for any atom.

(b) At zero pressure $\partial \varepsilon / \partial \xi=0$ so the slope of the curve for a neutral atom confined by a spherical piston tends to zero as the $(1,1)$ point is approached. This rule does not apply to compression by variation of nuclear charge because, if nuclear charge could vary freely, there would be no equilibrium at the $(1,1)$ point.

(c) For atoms confined by a spherical piston, there is a radius within which $E_{B}^{p}$ and therefore $\varepsilon$ change sign and so the curves cross the abscissa. This corresponds to delocalisation: the outermost electron is not bound by the atomic potential any more, but only by the spherical piston.

(d) For atoms compressed by an increase in nuclear charge, the ionisation potential increases with $Z$ : as the atom is compressed, its binding energy increases so the $(\xi, \varepsilon)$ curve veers upwards rather than downwards and never crosses the abscissa. This distinguishes between internal and external compression.

(e) Atoms can be dilated as well as compressed. This is achieved either by a reduction of nuclear charge or by confinement in an attractive shell [33]. Whatever the mechanism, there is never an increase in ionisation potential on dilation, hence a forbidden region for $\xi>1, \varepsilon>1$ in the $(\xi, \varepsilon)$ plane. Although converged solutions in the DiracFock code were not obtained for dilation by an attractive sphere, their shape can be deduced: branches of corresponding curves must be continuous at the $(1,1)$ point and the absent branch will clearly veer downwards to cross the abscissa when the atom is ionised. 
(f) The real physical pressure

$$
P=\eta(\varepsilon-1) /(\xi-1) .
$$

As already noted, most of the variation between atoms is in the factor $\eta$, which distinguishes between "soft" atoms (large alkalis e.g. Cs or Fr) and "hard" atoms (e.g. $\mathrm{He})$.

(g) Scaled $(\xi, \varepsilon)$ curves differ from one atom to another mainly by a slight rotation about $(1,1)$ as the pivot. This is enough to encompass nearly all elements in the Periodic Table (anticlockwise rotation for increasing hardness).

(h) Discontinuities due to orbital collapse [48] are of atomic origin even for solids: once orbital collapse is induced by an external pressure, the orbital involved is screened from the field of the solid and its atomicity, dramatically increased, which is why atomic structure calculations are successful in describing a wide range of orbital collapse phenomena in solids and compounds [49-54].

\subsection{Confinement resonances}

These considerations led the authors of reference [55] to consider and classify three different kinds of resonance which will appear in general for an atom confined inside a cavity of quantum size when the cavity is a real one, i.e. a hollow molecular cavity with penetrable walls and its own intrinsic spectrum.

Broadly, they are of three kinds:

(a) resonances of the confined atom modified by the presence of the confining cavity (atomic resonances);

(b) resonances intrinsic to the cavity, modified by the presence of the endohedral or confined atom (confinement resonances);

(c) molecular resonances which are present neither in the spectrum of the free atom nor in that of the empty cavity, but which appear only as a result of interactions between the atom and the cavity walls.

Usually, these different types of resonance appear in completely different energy ranges. If so, then a simple model in which they are not all included may be sufficient to treat the problem. However, it is also possible for resonances of different types to become degenerate and overlap in energy. This is especially the case for types (a) and (b). If this occurs, then a number of theoretical examples (e.g. [56]) show that the resonances will interact and exhibit Wigner avoided crossings [57], because they belong to the same quantum system.

Cavity resonances tend to be narrow and will, in this case, appear as intruder states within the modified atomic spectrum of the confined atom. However, while this is generally true, it must be remembered that the atom confined within a spherical molecule is not necessarily a rigid system and may well "rattle around" inside it. If there is such movement, then the observed spectrum will depend on which electron in the combined system is photo-excited and which part of the system it belongs to. Under these conditions, to observe confinement resonances embedded in an atomic spectrum the best situation is when the confined atom is a "tight fit" within the confining cavity, as occurs for Xe within the $\mathrm{C}_{60}$ fullerene. It is then more difficult for phase cancellations due to relative motion to occur and "smear out" any resonances. This may explain why, after the initial prediction of confinement resonances in 2000 [55], it took ten years for the first experimental report describing the effect [58] to appear. An alternative to enhance their visibility might be very short pulse excitation/detection. However, experiments on the spectroscopy of metallofullerenes remain very challenging because of the very low density of available targets and the low count rate achieved in detection. Probably, this will remain the case until it becomes possible to hold metallofullerenes in traps for more detailed spectroscopic investigation.

\subsection{Practical applications of atomic compression}

Although Sommerfeld and Welker had difficulty finding applications for their theory, the situation has changed considerably in the meantime. Atoms in zeolites [19], in quantum dots [59], in solids treated through the Watson sphere [60], in clusters [61] or superdense matter [62], in metallofullerenes [63] or in helium bubbles formed by escaping alpha particles in the steel walls of nuclear reactors [64] provide plenty of examples relevant to quantum confinement.

Much of the present review has concentrated on various forms of pressure applied to transition elements and rare-earths, because they are particularly "soft" and display interesting anomalies such as valence fluctuations and transitions between localised and itinerant behaviour. Their most important application today involves energy storage materials, either for so-called "rocking chair" batteries or for the storage of hydrogen in connection with the propulsion of vehicles. As previously commented, elements near the Smith and Kmetko ridge act as good catalysts and hydrogen storage materials. This is again because their outer wavefunctions are very flexible which allows them to adapt easily in size to changes in their immediate environment or external pressure.

Although an electric motor is extremely efficient (much more so than any thermal motor) the weak link is the storage of electrical energy. The propulsion of an electric car is therefore very dependent on the performance of the "rocking chair" batteries used to store and recover energy. The most efficient (power per weight) charge carrier in a battery is the Lithium ion. There is no better choice in the Periodic Table for this application. However, it poses a number of problems. The first is the choice of electrodes. The metal itself cannot be used. Unfortunately, hot lithium ignites spontaneously when in contact with air, which could result in fires or explosions. So, for a reversible $\mathrm{Li}+$ ion battery, it is necessary to develop materials into which the $\mathrm{Li}+$ ion can be implanted and stored without any possible contact with air so that the battery is safe. Finding suitable materials is more difficult the higher the current one draws from the battery, the greater the number of charging/discharging cycles and the greater the charge per cycle one wishes to store. Many different solutions have been tried, ranging from layered oxides to solid compounds with open spinelle structures, 
to polymers or even to fullerenes. This branch of applied research is both competitive and secretive because of its economic implications. Nonetheless, some general points can be made.

For solid electrodes (either layered oxides or open crystalline structures, favouring the free circulation of $\mathrm{Li}+$ ions), implantation and storage of the ions should be fully reversible. This requires that the lattice undergo polaronic distortion on lithiation but no recrystallization, because lattice recrystallization (a phase transition) during the storage and recovery process would result in energy loss and the properties of the material might change permanently or weaken the electrodes mechanically.

The need for only a single phase in the redox cycle was first recognised by Kosawa [65]. Thus, the emphasis is on a natural "atomic elasticity" which allows bonds to become distended reversibly and easily recoverable ion storage to occur. That is why many electrode materials favour the inclusion of metals from Smith and Kmetko's table which, as we have seen, are anomalously soft. Indeed, tables of "softness" for these atoms can be drawn up from ab initio atomic calculations. They can serve as an initial guide to determine which atoms possess useful properties for inclusion in electrode materials.

Similar considerations apply to hydrogen storage. For example "misch metal", a porous material which contains a high proportion of lanthanum provides a convenient adsorber of hydrogen which can be recovered reversibly by heating. Likewise, surfaces coated with $\mathrm{CeO}$ are good adsorbers of hydrogen. The process of storage and recovery without re-crystallisation is referred to as topotactic insertion. What makes it possible is again the "flexibility" of the atomic wavefunctions. The underlying atomic physics is discussed in [66].

\section{Conclusion}

Although the number of electrons in a neutral atom is fixed, its size, electronic configuration and chemical behaviour are not inalterable. They depend on the immediate environment of the atom in many interesting ways, or on the boundary conditions which are imposed theoretically when studying atomic models.

This approach to atomic physics is perhaps counterintuitive since there is a long established tradition in the subject of seeking the perfectly undisturbed, isolated atom in the purest state possible. In reality, however, atoms interact with surfaces, cavities, external fields as well as each other and the pressure effects described in the present review are in some ways the simplest examples of the atomic response to external perturbations.

From another angle (the connection to Thermodynamics) pressure effects explore the second term in the relation for internal energy (Eq. (3) above), while the first term, containing entropy and temperature) is left untouched. It is convenient in atomic physics to use zero-temperature models, so that the entropy (related to the number of microstates) can also be omitted and the consequences of Boltzmann's $H$ theorem [67] need not be considered, at least in the first instance. This means that atomic equations (such as the Hartree-Fock, Dirac-Fock, ThomasFermi or Density-Functional methods) are not as complete as the Ideal Gas Law, because they do not include any explicit temperature depen-dence. If we had a more complete quantum equation involving also the temperature, it could serve as the equation of state of a medium consisting of the atom inside an enclosure confined by a piston in exactly the same way as the equation of state of an ideal gas and would allow a new kind of thermodynamics to be developed, with isothermal and adiabatic compression and dilation cycles.

It is interesting that a thermodynamic model of a very primitive form (the "two-level" atom in equilibrium with a Black-Body radiation field at the temperature $T$ ) is basically how the Einstein-Milne rate equations were obtained $[7,8]$. The two-level atomic model, however, is far too simplistic: reference [2] above, on page 627 provides a simple description of a two-level system in thermodynamic equilibrium with a temperature bath. For such a system, increasing the temperature beyond the saturation point induces no further change in relative populations. The two-level system has therefore very limited relevance in the present context and, of course, contains no explicit connection to atomic compression. The Thomas-Fermi model provides a straightforward basis for counting the number of microstates, but still includes only those within the "Fermi sphere" with no temperature effects near its surface. There is a clear need to extend and combine existing models in order to achieve a "microscopic thermodynamic theory" of atoms, including both pressure and temperature effects at the same level around a single equation of state.

The Thomas-Fermi model and the Fermi-Thomas-Dirac model (with exchange) have both been used to derive equations of state for matter at very high pressures and various temperatures [68] yielding tabulated numerical solutions for specific cases. When exchange is included, a perturbative approach which includes the temperature effects additively is needed, but with the simpler ThomasFermi model, an exact formulation is possible. The effect of including temperature is to alter the charge distribution of electrons in the atom, because the simplest model at $T=0$ fills all the states up to the maximum momentum while leaving all the others empty. Substituting a FermiDirac distribution into Poisson's equation to derive a generalised Thomas-Fermi equation as hinted at in Section 2.1 above does yield an equation of state which can be integrated numerically, assuming a definite substance. However, the problem with approaches based on the statistical model of Thomas and Fermi is of course that the shell structure of the atom is not included correctly as, for example, in the Hartree-Fock model.

Finally, regarding applications, very high temperatures and pressures are encountered in stellar atmospheres. Likewise, extreme situations of high-pressure waves arise in laser-driven fusion experiments to which some of the conclusions described in the present paper about shell-filling under compression might well prove relevant. 


\section{Author contribution statement}

This is a single author paper. I am grateful to the referee for useful comments on the original draft.

Publisher's Note The EPJ Publishers remain neutral with regard to jurisdictional claims in published maps and institutional affiliations.

Open Access This is an open access article distributed under the terms of the Creative Commons Attribution License (https://creativecommons.org/licenses/by/4.0/), which permits unrestricted use, distribution, and reproduction in any medium, provided the original work is properly cited.

\section{References}

1. A. Sommerfeld, H. Welker, Ann. Phys. 32, 56 (1938)

2. C. Cohen-Tannoudji, B. Diu, F. Laloë, in Quantum Mechanics (Hermann, Paris, 1977), Vol. 1, pp. 624-626

3. P.A.M. Dirac, in The Principles of Quantum Mechanics (Clarendon Press, Oxford, 1981), p. 314

4. Bl. Pascal, in Les Pensées, Chez Lefebvre, Libraire rue de l'Epernon $N^{\circ} 6$, Paris Published in 1826 pages 74 to 78 (1660)

5. M. Zemansky, Heat and Thermodynamics (McGraw Hill Book Co, New York, 1943)

6. W. Pauli, Zeitschrift für Physik. 31, 765 (1925)

7. A. Einstein, Zeit für Phys. Z. 18, 121 (1917)

8. E.A. Milne, MNRAS 84, 111 (1924)

9. L.H. Thomas, Math. Proc. Camb. Philos. Soc 23, 542 (1927)

10. E. Fermi, Zeitschrift für Physik 48, 73 (1928)

11. P.A.M. Dirac, Math. Proc. Camb. Philos. Soc 26, 376 (1930)

12. E.H. Lieb, B. Simon, Adv. Math. 23, 22 (1930)

13. N.H. March, Origins - The Thomas-Fermi Theory, in Theory of The Inhomogeneous Electron Gas, edited by S. Lundqvist, N.H. March (Plenum Press, London, 1983)

14. J.-P. Connerade, V.K. Dolmatov, J. Phys. B: At., Mol. Opt. Phys. 31, 3557 (1998)

15. B.H. Bransden, C.J. Joachain, in Physics of Atoms and Molecules (Longman London, New York, 1983), p. 313

16. S.R. De Groot, C.A. Ten Seldam, Physica, 12, 669 (1946)

17. N. Aquino, Adv. Quantum Chem. 57, 123 (2009)

18. A. Michels, J. de Boer, A. Bijl, Physica 4, 981 (1938)

19. W. Jaskólski, Phys. Rep. 27, 1 (1996)

20. J.L. Smith, E.A. Kmetko, J. Less Common Metals 90, 83 (1983)

21. A.R. Mackintosh, Physica B+C, 130, 112 (1985)

22. E. Fermi, Quantentheorie und Chemie, edited by $\mathrm{H}$. Falkenhagen (Leipziger vorträge Published by S. HirzelVerlag, Leipzig, 1928)

23. N. Bohr, in The Theory of Spectra and Atomic Constitution (Cambridge University Press, Cambridge UK, 1922), p. 126

24. G. Herzberg, Atomic Spectra and Atomic Structure (Dover Publications Inc, 31 East 2nd Street, Mineola New York 11501, 1944)

25. M. Goeppert-Mayer, Phys. Rev. 60, 184 (1941)

26. D.C. Griffin, K.L. Andrew, R.D. Cowan, Phys. Rev. 177, $62(1969)$
27. J.-P. Connerade, Highly Excited Atoms, in Cambridge Monographs on Atomic, Molecular and Chemical Physics (Cambridge University Press, Cambridge UK, 1998), Chapt. 5

28. J.-P. Connerade, J. Phys. B: At., Mol. Opt. Phys. 24, L109 (1991)

29. J.-P. Connerade, V.K. Dolmatov, P.A. Lakshmi, J. Phys. B: At., Mol. Opt. Phys. 33, 251 (2000)

30. J.-P. Connerade, J.-M. Esteva, R.C. Karnatak, Giant Resonances in Atoms Molecules and Solids, in Les Houches NATO ASI Series B Physics (Plenum Press, New York and London, 1987), Vol. 151

31. N. Levinson, Danske Vidensk Selsk K Mat Fys Medd 25, (1949)

32. J. Kellendonk, S. Richard, The topological meaning of Levinson's theorem (2007)

33. J.-P. Connerade, R. Semaoune, J. Phys. B: At., Mol. Opt. Phys. 33, 869 (2000)

34. V. Alonso, S. De Vincenzo, L. Mondino, Eur. J. Phys. 18, 315 (1997)

35. L.E. Klintberg, S.K. Goh, S. Kasahara, Y. Nakai, K. Ishida, M. Sutherland, T. Shibauchi, Y. Matsuda, T. Terashima, J. Phys. Soc. Jpn. 79, 123706 (2010)

36. H. Hellmann, in Einführung in die Quantenchemie (Leipzig, Franz Deuticke, 1937), p. 285

37. R.P. Feynman, Phys. Rev. 56, 340 (1939)

38. W. Nieman, W. Malzfeld, P. Rabe, R. Haensel, M. Lübcke, Phys. Rev. B35, 1099 (1987)

39. J.-P. Connerade, J. Phys. C. Solid State 15, L367 (1982)

40. J.M. Lawrence, P.S. Riseborough, R.D. Parks, Rep. Prog. Phys. 44, 1 (1981)

41. R. Ramirez, L.M. Falcov, J.C. Kimball, Phys. Rev. B 2, 3383 (1970)

42. F.D.M. Haldane, Phys. Rev. B 15, 281 (1977)

43. F.D.M. Haldane, Phys. Rev. B 15, 2477 (1977)

44. D.J. Khomskii, A.N. Kocharjan, Solid State Commun. 18, 985 (1976)

45. J.-P. Connerade, P. Kengkan, P. Anantha Lakshmi, R. Semaoune, J. Phys. B: At., Mol. Opt. Phys. 33, L847 (2000)

46. K.G. Dyall, I.P. Grant, C.T. Johnson, F.A. Parpia, E.P. Plummer, Comput. Phys. Commun. 55, 425 (1989)

47. J.-P. Connerade, R. Semaoune, J. Phys. B: At., Mol. Opt. Phys. 33, 3467 (2000)

48. J.-P. Connerade, P. Kengkan, Atomic Confinement, Electron Scattering, Physics of Atoms and Molecules, edited by C.T. Whelan, N.J. Mason (Springer, Boston MA, 2005)

49. J.-P. Connerade, J. Less Common Metals 93171 (1983)

50. M. Schlüter, C.M. Varma, Helv. Physica Acta 56, 147 (1983)

51. C. Bansal, V. Srinivasan, Solid State Commun. 51, 781 (1984)

52. R. Ruus, in Dissertationes Physicae Universitatis Tartuensis (1999), Vol. 31

53. J.C.A. Boeyens, J. Chem. Soc., Faraday Trans. 90, 3377 (1994)

54. J. Olivier-Fourcade, J.C. Jumas, J.-P. Connerade, J. Chem. Phys. 97, 6801 (1999)

55. J.P. Connerade, V.K. Dolmatov, S.T. Manson, J. Phys. B: At., Mol. Opt. Phys. 33, 2279 (2000)

56. J.-P. Connerade, V.K. Dolmatov, P.A. Lakshmi, S.T. Manson, J. Phys. B: At., Mol. Opt. Phys. 32, L239 (1999)

57. J. von Neumann, E.P. Wigner, Physikalische Zeitschrift 30, 465 (1929) 
58. D. Kilcoyne, A. Aguilar, A. Müller, A.S. Schippers, C. Cisneros, G. Alna'washi, N. Aryal, K. Baral, D. Esteves, C. Thomas, R. Phaneuf, Phys. Rev. Lett. 105, 213001 (2010)

59. L.G. Gerchikov, V.F. Masterov, Appl. Phys. Lett. 73, 532 (1998)

60. R.E. Watson, Phys. Rev. 111, 1108 (1958)

61. M. Velegrakis, C. Lüder, Chem. Phys. Lett. 223, 139 (1994)

62. R.M. More, Adv. At. Mol. Opt. Phy. 21, 305 (1985)

63. L. Moro, R.S. Rouff, C.H. Becker, V.C. Lorentz, R. Malhotra, J. Chem. Phys. 97, 6801 (1993)

64. D.W. Essex, N. Pyper, C.T. Whelan, Calculations of the He Is2Is2p energy shift observed in STEM experiments on helium bubbles in irradiated materials, in Electron
Microscopy and Analysis 1999 (Institute of Physics Electron Microscopy and Analysis Group Conference, University of Sheffield, 1999), p. 187

65. A. Kozawa, in Applications of Solid Electrolytes, edited by T. Takahashi, A. Kozawa (JEC Press Inc. Cleveland Ohio, 1980), p. 23

66. J.-P. Connerade, J. Alloys Compd. 255, 79 (1997)

67. C. Kittel, in Elementary statistical physics (John Wiley and Sons Inc, New York, 1958), p. 171

68. R.P. Feynman, N. Metropolis, E. Teller, Phys. Rev. 75, 1561 (1949)

69. M. Rahm, R. Cammi, N.W. Ashcroft, R. Hoffmann, J. Am. Chem. Soc. 141, 10253 (2019) 